Original article

\title{
Assessing the consistency of liver fibrosis results obtained through biopsy and transient elastography in patients suffering from chronic hepatitis $C$
}

\author{
Agnieszka Pokora, Sławomir Kiciak, Krzysztof Tomasiewicz \\ Department of Infectious Diseases, Medical University of Lublin, Poland
}

\begin{abstract}
Until recently, core-needle liver biopsy was used as the gold standard in chronic hepatitis C diagnostics. Information on the inflammatory activity grade and the staging of liver fibrosis or steatosis, obtained through biopsy, constituted an indispensable element in the process of determining patients' eligibility for antiviral treatment. The histological profile of the samples examined was decisive in determining the time for commencing treatment. Given that this procedure involves the risk of complications and temporarily reduces patient living comfort, it was deemed necessary to search for other ways to assess liver fibrosis. Transient elastography is a non-invasive alternative to liver biopsy. The current chronic hepatitis $C$ treatment programme provides the possibility to perform transient elastography instead of liver biopsy. The test result is expressed in $\mathrm{kPa}$. However, liver biopsy is still recommended in certain cases of unclear aetiology, or if discrepancies are found between the elastography results and patient clinical condition. The aim of this study was to compare two methods of liver fibrosis assessment in terms of consistency of results, and to analyse any inconsistent results.
\end{abstract}

Key words: chronic hepatitis C, liver biopsy, transient elastography, non-invasive, fibrosis.

Address for correspondence

Agnieszka Pokora, Department of Infectious Diseases, Medical University of Lublin, 16 Staszica St., 20-081 Lublin, Poland, e-mail: agniecha811@gmail.com

\section{Introduction}

For many years liver biopsy has been used as the gold standard to assess liver fibrosis in patients suffering from chronic hepatitis $\mathrm{C}$. The histological examination of liver tissue constituted grounds for postponing antiviral treatment, or for its immediate commencement, with a view to avoiding the adverse consequences of hepatitis $\mathrm{C}$ virus (HCV) infection in the short perspective. The METAVIR scoring system is the most commonly used one for assessing inflammatory activity grade and the staging of fibrosis. These scores are defined as follows:

- stages of fibrosis (F): F0 - no fibrosis, F1 - portal fibrosis without septa, F2 - portal fibrosis with rare septa, F3 - numerous septa without cirrhosis, F4 cirrhosis;
- grade for activity (A): A0 - no histologic necroinflammatory activity, A1 - minimal activity, A2 moderate activity, A3 - severe activity.

A biopsy involves the risk of such complications as pain, asymptomatic bleeding (subcapsular and intracapsular haematomas) and bleeding into the peritoneal cavity. Also, the risk of an accidental puncture of other body organs, including mainly the lungs (resulting in pneumothorax, aerodermectasia or pleural effusion), the kidneys, the large intestine, and the gall bladder, cannot be eliminated. Very often this procedure can cause bleeding into the biliary tract, which manifests as hepatitis, biliary colic or tarry stools. Contra-indications to liver biopsy include a lack of cooperation with the patient, extrahepatic biliary tract obstruction, and bacterial bile-duct inflammation (a relative contra-indication; the collection of a biopsy specimen 
Table 1. Liver stiffness cut-offs in chronic liver diseases according to Castera et al.

\begin{tabular}{cccc}
\hline F0-F1 & F2 & F3 & F4 \\
\hline $2.5-7 \mathrm{kPa}$ & $7.1-9.5 \mathrm{kPa}$ & $9.6-12.5 \mathrm{kPa}$ & $>12.5 \mathrm{kPa}$ \\
\hline
\end{tabular}

and its bacteriological analysis [culture] can help to determine the disease etiology, e.g. unexplained fever), coagulation disorders (however, there are contradictory opinions as to the contra-indications for biopsy in the case of coagulation system disorders; it is recommended that the coagulation ratios be checked before any biopsy, and the procedure is considered safe when performed within 24 hours after mitigating any coagulation-factor deficiencies). In every case it is advisable to consider both the benefits from biopsy and the risk of complications, including patient's death [1].

Transient elastography as a non-invasive method involves no risk of complications, is performed in out-patient conditions, and does not reduce patients' living comfort. As it is a repeatable procedure, it increases the frequency of fibrosis monitoring and examination regularity, providing an opportunity to analyse the fibrosis-process dynamics $[2,3]$. Indications for this proce-

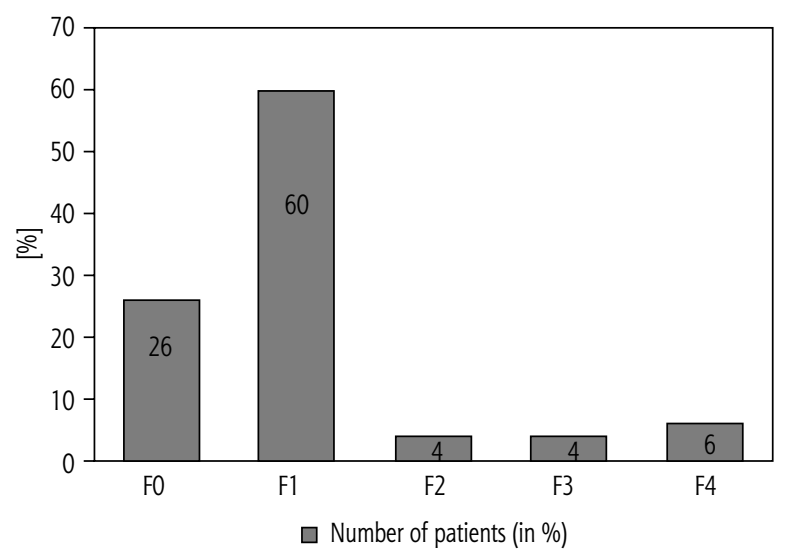

Fig 1. Fibrosis assessment through liver biopsy

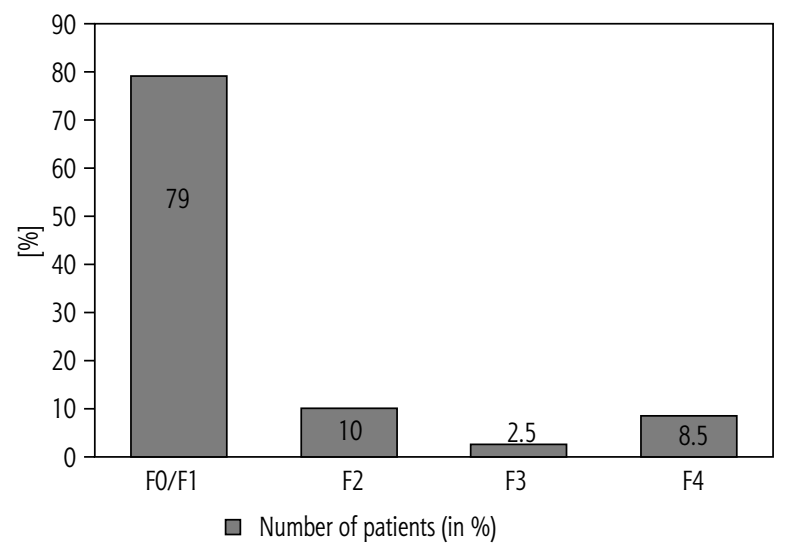

Fig. 2. Fibrosis assessment through transient elastography dure include chronic hepatitis $\mathrm{B}$ and $\mathrm{C}$, non-alcoholic fatty liver disease (NAFLD), alcoholic liver disease, primary biliary cirrhosis and primary sclerosing cholangitis [2]. The person performing the procedure reads out its result immediately after the examination [4]. The probe induces an elastic (acoustic) wave that moves towards the liver. It is assumed that ten measurements at a success rate of $>60 \%: \mathrm{IQR} / \mathrm{M}<0.3$ deliver a valid result. The wave propagation speed is assessed in the liver parenchyma area located at 2.5 to $6.5 \mathrm{~cm}$ from the skin surface (the skin capsular distance) [5]. The measurement covers a cylinder with a diameter of $1 \mathrm{~cm}$ and length of $4 \mathrm{~cm}$ (100 times more than the biopsy specimen) [2].

The overall values fall within the range of 3.3 to $75 \mathrm{kPa}$. These values have been converted into corresponding degrees of the METAVIR scoring system, using the cut-offs proposed by Castera et al. (Table 1) [6].

Contraindications to transient elastography include pregnancy, ascites, an implanted cardiac pacemaker and a lack of cooperation with the patient.

The aim of this study was to compare the two methods of assessing liver fibrosis in terms of consistency of results, and to analyse any inconsistent results.

\section{Material and methods}

The analysis included 80 patients with hepatitis C undergoing the process of determining eligibility for antiviral treatment. All the patients underwent coreneedle liver biopsy. Its results were assessed by one pathologist using the METAVIR scoring system. Transient elastography was performed on the same day (prior to liver biopsy). Measurements of liver stiffness using a FibroScan ${ }^{\circledast} \mathrm{M}$ probe were taken by one analyst.

The study group consisted of 53 women (66\% of patients) and 27 men (34\%). The average age was 44 years (age from 21 to 67). The calculation was performed us-

Table 2. A compilation of inconsistent liver fibrosis assessment results obtained through biopsy and transient elastography

\begin{tabular}{cccc}
\hline Sex & $\begin{array}{c}\text { METAVIR } \\
\text { Biopsy }\end{array}$ & $\begin{array}{c}\text { kPa } \\
\text { FibroScan }\end{array}$ & $\begin{array}{c}\text { METAVIR } \\
\text { Fibroscan }\end{array}$ \\
\hline$F$ & A2F1 & 9.4 & F2 \\
\hline$M$ & A2F1 & 7.6 & F2 \\
\hline$F$ & A3F1 & 8.8 & F2 \\
\hline$F$ & A3F3 & 17.1 & F4 \\
\hline$F$ & A3F3 & 16.0 & F4 \\
\hline M & A2F3 & 16.4 & F4 \\
\hline
\end{tabular}


ing the IBM SPSS 23.0 and R 3.2.0 softwares. For the assessment of conformity between distributions and normal distribution the Kolmogorov-Smirnov test was used. In order to assess the correlation between quantitative variables, Spearman's correlation index was used. The results were presented graphically using scatter graphs. The statistical significance limit of $p<0.05$ was adopted. The diagnostic test was assessed using a contingency table. The following variables were calculated: sensitivity, specificity, and the probability of true positive, false positive, true negative and false negative results.

\section{Results}

The fibrosis assessment results were consistent in 74 out of 80 cases, which accounted for $92.5 \%$ of all results gathered. When assessing fibrosis through biopsy, the following percentage distribution was demonstrated: F0 - 26\%, F1 - 60\%, F2 - 4\%, F3 - 4\%, and F4 - 6\% (Fig. 1). The results of fibrosis assessments through transient elastography were as follows: F0/F1 - 79\%, F2 - 10\%, F3 - 2.5\%, and F4 - 8.5\% (Fig. 2).

A statistically significant positive correlation between the variables was identified $(\rho=0.657 ; p=0.000)$. The correlation coefficient equalled 0.657 . The sensitivity of elastography, as compared to biopsy, was 0.7 , and the specificity was 1 . The probability of a true positive test result: the probability of a false positive test result: 0 ; the probability of a true negative test result: 0.928571 ; the probability of a false negative test result: 0.071429 .

In the case of 6 patients the fibrosis stage identified in transient elastography was higher than that revealed by liver biopsy.

\section{Conclusions}

92.5\% consistency between the two liver fibrosis assessment methods was demonstrated. A statistically significant positive correlation between the variables was identified $(\rho=0.657 ; p=0.000)$.

In the case of 6 patients whose results of biopsy and transient elastography were inconsistent, it was found that the fibrosis stage in transient elastography was higher than that revealed by biopsy, which would have no influence on treatment postponement (Table 2).

Among all the patients in the aforementioned group, the inflammatory activity grade in biopsy was A2 or A3. In this group alanine aminotransferase was above 100 IU/l. Inflammation was the likely cause of the increased level of fibrosis, as compared to biopsy result $[3,4,7]$.

Transient elastography may be used to assess liver fibrosis in patients with chronic hepatitis C [8-14].

\section{Disclosure}

Authors report no conflict of interest.

\section{References}

1. Kierzkowska I. Wytyczne do wykonania biopsji wątroby w praktyce klinicznej. Medycyna Praktyczna 2000; 7-8: 145-160.

2. De Lédingen V, Vergniol J. Transient elastography for the diagnosis of liver fibrosis. Expert Rev Med Devices 2010; 7: 811-823.

3. Kumagai E, Korenaga K, Korenaga M, et al. Appropriate use of virtual touch quantification and FibroScan ${ }^{\circledast} \mathrm{M}$ and XL probes according to the skin capsular distance. J Gastroenterol 2015; 13: 1-10.

4. Scott DR, Levy MT. Liver transient elastography (FIbroScan): a place in the management algorithms of chronic viral hepatitis. Antivir Ther 2010; 15: 1-11.

5. Wong GL. Prediction of fibrosis progression in chronic viral hepatitis. Clin Mol Hepatol 2014; 20: 228-236.

6. Castera L, Bendossa P. How to assess liver fibrosis in chronic hepatitis C: serum markers or transient elastography vs. liver biopsy? Liver Int 2011; 31: 13-17.

7. Chan HL, Wong GL, Choi PC, et al. Alanine aminotrasferase-based algorithms of liver stiffness measurement by transient elastography (Fibroscan) for liver fibrosis in chronic hepatitis B. J Viral Hepatitis 2009; 16: 36-44.

8. Castera L, Forns X, ALberti A. Non-invasive evaluation of liver fibrosis using transient elastography. J Hepatol 2008; 48: 835-847.

9. Wong GL. Update of liver fibrosis and steatosis with transient elastography (Fibroscan). Gastroenterol Rep (Oxf) 2013; 1: 19-26.

10. Afdhal NH, Bacon BR, Patel K, et al. Accuracy of Fibroscan, compared with histology, in analysis of liver fibrosis in patients with hepatitis B or C: a United States multicenter study. Clin Gastroenterol Hepatol 2015; 13: 772-779.

11. Steadman R, Myers RP, Leggett L, et al. A health technology assessment of transient elastography in adult liver disease. Can J Gastroenterol 2013; 27: 149-158.

12. Stevenson M, Lloyd-Jones M, Morgan MY, et al. Non invasive diagnostic assessment tools for the detection of liver fibrosis in patients with suspected alcohol-related liver disease: a systematic review and economic evaluation. Health Technol Assess 2012; 16: 1-174.

13. Castera L, Bendossa P. How to assess liver fibrosis in chronic hepatitis C: serum markers or transient elastography vs.liver biopsy? Liver Int 2011; 31: 13-17.

14. Murtagh J, Foerster V. Transient elastography (Fibroscan) for non-invasive assessment of liver fibrosis. Issues Emerg Health Technol 2006; 90: 1-4. 